\title{
APPROXIMATION WITH NORMS DEFINED BY DERIVATIONS
}

\author{
J. M. BRIGGS \\ (Received 23 May 1972; revised 9 January 1973) \\ Communicated by J. B. Miller
}

A linear mapping $D$ of the algebra of polynomial functions $P[0,1]$ into the algebra of all continuous complex-valued functions $C[0,1]$ is called a derivation provided $D(f g)=f D(g)+g D(f)$ for all polynomials $f$ and $g$. The derivations of $P[0,1]$ into $C[0,1]$ are easily seen to be all mappings of the form $D_{w}$ where $w$ is a continuous function on $[0,1]$ and $D_{w}(f)=w f^{\prime}\left(f^{\prime}\right.$ denotes the ordinary derivative of $f$ ). In fact, $w=D(x)$ where $x$ is the coordinate function. Let $D_{w}$ be such a derivation, and let $\|\cdot\|$ denote the supremum norm on $C[0,1]$. Then $D_{w}$ gives rise to an algebra norm $\|\cdot\|_{w}$ on $P[0,1]$ defined by

$$
\|f\|_{w}=\|f\|+\left\|D_{w}(f)\right\|=\|f\|+\left\|w f^{\prime}\right\| \text { for } f \in P[0,1] .
$$

In this paper we study the algebra of all continuous functions on $[0,1]$ which are $\|\cdot\|_{w^{-}}$-approximable by polynomials; that is, those functions which are pointwise limits of $\|\cdot\|_{w}$-Cauchy sequences of polynomials. Let $C^{1}(w)$ denote the algebra of all such functions. For comparison purposes, we define two other algebras of functions. For $w \in C[0,1]$ let $\mathscr{Z}(w)$ denote the zero set of $w$. Let $C_{w}^{1}$ denote the subalgebra of $C[0,1]$ consisting of all $f$ such that $(\mathrm{i}) f^{\prime}(y)$ exists for each $y \in[0,1] \backslash \mathscr{Z}(w)$, and (ii) the function $w f^{\prime}$ is continuous on $[0,1]$ where $\left(w f^{\prime}\right)(y)=0$ if $y \in \mathscr{Z}(w),\left(w f^{\prime}\right)(y)=w(y) f^{\prime}(y)$ if $y \in[0,1] \backslash \mathscr{Z}(w)$. Finally, let $A C_{w}$ be the subalgebra of $C_{w}^{1}$ consisting of absolutely continuous functions.

The following are the main results of this paper. Two algebras $C^{1}\left(w_{1}\right)$ and $C^{1}\left(w_{2}\right)$ are equal if and only if there exists a bounded function $h$ on $[0,1]$ which is bounded away from zero such that $w_{2}=h w_{1}$. The method of approximation described in this paper generalizes both uniform approximation of continuous functions and the familiar method of approximation of once continuously differentiable functions, since $C^{1}(w)=C[0,1]$ if and only if $w \equiv 0$, and $C^{1}(w)=C^{1}[0,1]$ if and only if $w$ is never zero. As the following results indicate, the zero set of $w$ plays an important role in what can be approximated. There 
exists a non-constant function $f$ such that $w f^{\prime}=0$ if and only if $\mathscr{Z}(w)$ is uncountable; $C^{1}(w)=A C_{w}$ if and only if $1 / w \in L^{1}[0,1]$. If the boundary of $\mathscr{Z}(w)$ is countable, then $C^{1}(w)=C_{w}^{1}$. Finally, as an example shows, if $\mathscr{Z}(w)$ is not suitably simple, then we should not expect that $C^{1}(w)$ will equal $C_{w}^{\mathbf{1}}$.

The author expresses appreciation for the suggestions made by the referees in reading this paper; the present statement and proof of Theorem 6 are due to them.

\section{Preliminaries}

In this paper we use the general theory of Banach algebras for two purposes: to obtain norm estimates and to use localization. Let $A$ be a semi-simple, commutative Banach algebra over $C$ with identity which we consider as an algebra of complex-valued continuous functions on its maximal ideal space $\mathscr{M}(A)$ via the Gelfand representation. $A$ is called regular provided that for each closed set $F$ in $\mathscr{M}(A)$ and point $p$ not in $F$, there exists an element $f \in A$ such that $f(p)=1$ and $f \mid F=0$ (where $\mid$ denotes the restriction). If $g$ is a continuous function on $\mathscr{M}(A)$ and $p \in \mathscr{M}(A)$, we say that $g$ belongs locally to $A$ at $p$ provided there exists a neighborhood $U$ of $p$ and an element $f \in A$ such that $f|U=g| U$. It is well known that if $A$ is regular, then a continuous function $g$ belongs to $A$ if and only if $g$ belongs locally to $A$ at each poibt of $\mathrm{M}(A)$ (see, for instance, page 224 of Naimark (1964)). Another fact which will be useful gives a comparison between the topologies of a Banach algebra and its subalgebras. Let $A_{1}$ and $A_{2}$ be commutative Banach algebras with norms $\|\cdot\|_{1}$ and $\|\cdot\|_{2}$ respectively such that $A_{2}$ is semi-simple and $A_{1} \subseteq A_{2}$. Then there exists a constant $M$ such atht $\|a\|_{2}$ $\leqq M\|a\|_{1}$ for all $a \in A_{1}$. (This is a consequence of Theorem 2.5.17 of Rickart(1960).)

Let $A$ and $B$ be commutative algebras over $\mathbf{C}$ with the identity of $A$ contained in $B$ and $B \subseteq A$. A linear mapping $D$ of $B$ into $A$ is called a derivation if $D(f g)=f D(g)+g D(f)$ for all $f, g \in B$. Notice that since $B$ contains the identity, the kernel of $D$ must contain the constants. We say that $D$ is almost injective if $\operatorname{ker}(D)=\mathbf{C}$.

Let $C[0,1]$ denote the algebra of all continuous, complex-valued functions on $[0,1]$ with the supremum norm $\|\cdot\|$, and $C^{1}[0,1]$ the algebra of complexvalued, continuously differentiable functions on $[0,1]$ with the norm $\|\cdot\|_{1}$ defined by $\|f\|_{1}=\|f\|+\left\|f^{\prime}\right\|$ where $f^{\prime}$ denotes the derivative of $f$. Let $A C[0,1]$ denote the algebra of complex-valued, absolutely continuous functions on $[0,1]$ with the norm $\|\cdot\|^{\prime}$ defined by $\|f\|^{\prime}=\|f\|+\int_{0}^{1}\left|f^{\prime}\right|$. These three algebras are regular, semi-simple, commutative Banach algebras with identity having $[0,1]$ as their maximal ideal spaces and each containing the dense subalgebra $P[0,1]$ of polynomials. (See pages $300-303$ of Rickart (1960); also see Theorem 2 of Loy (1970).) 


\section{Derivations and Approximation}

The derivation $D_{w}$ of $P[0,1]$ into $C[0,1]$ has a natural extension $\tilde{D}_{w}$ to the algebra $C_{w}^{1}$ : for each $f \in C_{w}^{1}$, let $\tilde{D}_{w}(f)=w f^{\prime}$ as defined earlier. Then it is easy to see that $\tilde{D}_{w}$ is a derivation of $C_{w}^{1}$ into $C[0,1]$ which extends $D_{w}$. Define a norm $\|\cdot\|_{w}$ on $C_{w}^{1}$ by $\|f\|_{w}=\|f\|+\left\|\tilde{D}_{w}(f)\right\|=\|f\|+\left\|w f^{\prime}\right\|$. Since $\tilde{D}_{w}$ is a derivation, $\|\cdot\|_{w}$ is submultiplicative. Hence, $C_{w}^{1}$ is a normed algebra; furthermore, it is easily verified that it is a Banach algebra and that $D_{w}$ is a closed derivation of $C_{w}^{\mathbf{1}}$ into $C[0,1]$. Let $C^{1}(w)$ be the closure in $C_{w}^{1}$ of $P[0,1]$; that is, $C^{\mathbf{1}}(w)$ consists of those functions in $C[0,1]$ which can be approximated in this norm $\|\cdot\|_{w}$. The algebras $C_{w}^{1}$ and $C^{1}(w)$ give examples of algebras of derivable elements (see p. 310 of Loy (1970)). If we let $M=\max \{1,\|w\|\}$, then $\|p\| \leqq\|p\|_{w} \leqq M\|p\|_{1}$ for all $p \in P[0,1]$. The next theorem is a simple consequence of this inequality.

THEOREM 1. $C^{\mathbf{1}}[0,1] \subseteq C^{1}(w) \subseteq C_{w}^{1} \subseteq C[0,1]$.

Furthermore, each of these algebras is semi-simple and regular, and each has $[0,1]$ as its maximal ideal space.

Notice that $C^{1}(w)=C^{1}(|w|), C_{w}^{1}=C_{|w|}^{1}$, and $A C_{w}=A C_{|w|}$. Hence, when it is convenient for computing these algebras, we may assume that $w \geqq 0$. The remainder of this paper will be devoted to comparing and describing these algebras.

Lemma 2. If $C^{1}\left(w_{1}\right) \subseteq C^{1}\left(w_{2}\right)$, then $\mathscr{Z}\left(w_{1}\right) \subseteq \mathscr{Z}\left(w_{2}\right)$.

Proof. If there were a point $x_{0}$ in $\mathscr{Z}\left(w_{1}\right)$ but not in $\mathscr{Z}\left(w_{2}\right)$, then, because $x_{0}$ is not in $\mathscr{Z}\left(w_{2}\right)$, every function in $C^{1}\left(w_{2}\right)$ would be continuously differentiable in some neighborhood of $x_{0}$. We show that this leads to a contradiction if $C^{1}\left(w_{1}\right) \subseteq C^{1}\left(w_{2}\right)$. More generally, suppose that $f^{\prime}(x)$ exists for all $f$ in $C^{1}(w)$ but that $w(x)=0$. Since $f^{\prime}(x)=\lim \left(f\left(x_{n}\right)-f(x)\right) /\left(x_{n}-x\right)$ when $\lim x_{n}=x$, the uniform boundedness principle yields the existence of a constant $M$ such that $\left|f^{\prime}(x)\right| \leqq M\left(\|f\|+\left\|w f^{\prime}\right\|\right)$ for all $f$ in $C^{1}(w)$. Let $U$ be a neighborhood of $x$ for which $\sup _{v}|w|<1 / 2 M$. Then for all $f$ in $C^{1}[0,1]$ which are constant outside $U$, we have $\left|f^{\prime}(x)\right| \leqq M\|f\|+(1 / 2)\left\|f^{\prime}\right\|$; but it is easy to see that there are such $f$ with $f^{\prime}(x)=\left\|f^{\prime}\right\|$ and $\|f\|$ arbitrarily small, thus reaching a contradiction.

THEOREM 3. $C^{1}\left(w_{1}\right) \subseteq C^{1}\left(w_{2}\right)$ if and only if there exists a bounded function $h$ on $[0,1]$ such that $h \mid \mathscr{Z}\left(w_{1}\right)=1$ and $w_{2}=h w_{1}$.

Proof. Suppose that $w_{2}=h w_{1}$ where $h$ is bounded by $M \geqq 1$. If $p \in P[0,1]$, then $\|p\|_{w_{2}} \leqq M\|p\|_{w_{1}}$. Hence $C^{1}\left(w_{1}\right) \subseteq C^{1}\left(w_{2}\right)$.

Now suppose that $C^{1}\left(w_{1}\right) \subseteq C^{1}\left(w_{2}\right)$. By semisimplicity there exists a constant $M>1$ such that $\|f\|_{w_{2}} \leqq M\|f\|_{w_{1}}$ for all $f$ in $C^{1}[0,1]$. We claim that $w_{2} / w_{1}$ is bounded by $M$ outside $\mathscr{Z}\left(w_{1}\right)$. If not, there exists an interval $I$, disjoint from 
$\mathscr{Z}\left(w_{1}\right)$, such that $\left|w_{2} / w_{1}\right| \geqq N$ on $I$, where $N>M$. Then, for any $f$ in $C^{1}[0,1]$ which is constant outside $I$, we have

$$
\|f\|_{w_{2}}=\|f\|+\left\|w_{1}\left(w_{2} / w_{1}\right) f\right\| \geqq\|f\|+N\left\|w_{1} f^{\prime}\right\|
$$

and thus

or

$$
M\left(\|f\|+\left\|w_{1} f^{\prime}\right\|\right) \geqq\|f\|+N\left\|w_{1} f^{\prime}\right\|
$$

$$
\left\|w_{1} f^{\prime}\right\| \leqq(M-1) /(N-M)\|f\| \text { for all such } f \text {. }
$$

It then must be true that

$$
\left\|f^{\prime}\right\| \leqq(M-1) /\left((N-M) \min _{1}\left|w_{1}\right|\right)\|f\|=K\|f\|
$$

which is clearly impossible. Thus $w_{2} / w_{1}$ is bounded and the conclusion of the theorem follows since $\mathscr{Z}\left(w_{1}\right) \subseteq \mathscr{Z}\left(w_{2}\right)$.

Corollary 4. $C^{1}\left(w_{1}\right)=C^{1}\left(w_{2}\right)$ if and only if there exists a function $h$ on $[0,1]$ which is both bounded above and bounded away from zero such that $w_{2}=h w_{1}$.

Corollary 5. $C^{1}(w)=C[0,1]$ if and only if $w \equiv 0 ; C^{1}(w)=C^{1}[0,1]$ if and only if $w$ is never zero.

Before comparing the algebras $C^{1}(w), C_{w}^{1}$, and $A C_{w}$, we characterize when the derivation $\tilde{D}_{w}$ is almost injective.

THEOREM 6. $\tilde{D}_{w}$ is almost injective if and only if $\mathscr{Z}(w)$ is countable.

Proof. Suppose $\mathscr{Z}(w)$ is uncountable. Then it contains a perfect set $K$ with empty interior (see p. 228 of Sierpinski (1952)). Following Cantor, we can construct a nonconstant continuous function $f$ which is constant on each interval of the complement of $K$, and for this $f$, we have $w f^{\prime}=0$. Suppose now that $\mathscr{Z}(w)$ is countable. If $w f^{\prime}=0$, then $f$ is certainly constant on each interval of the complement of $\mathscr{Z}(w)$. Let $U$ be the set of all points $x$ such that $f$ is constant in some neighborhood of $x$. Then the complement of $U$ is a closed set without isolated points, since $f$ is continuous, and contained in $\mathscr{Z}(w)$. Hence this set is empty or uncountable; but since $\mathscr{Z}(w)$ is countable, it is empty. Thus $f$ is constant.

Our last task will be to describe what these algebras are in many cases.

LEMMA 7. $A C_{w} \subseteq C^{1}(w)$.

Proof. Let $g \in A C_{w}$ and $\varepsilon>0$. Since $g \in A C[0,1]$, there exists $f \in C^{1}[0,1]$ such that $\|f-g\|<\varepsilon / 4$ and $\int_{0}^{1}\left|f^{\prime}-g^{\prime}\right|<\varepsilon / 4$. Let $\delta$ be such that if $S \subseteq[0,1]$ and meas $(S)<\delta$, then $\int_{S}\left|f^{\prime}\right|+\left|g^{\prime}\right|<\varepsilon / 8$. Since $w$ and $w g^{\prime}$ are zero on $\mathscr{Z}(w)$, there exists an open neighborhood $U$ of $\mathscr{Z}(w)$ such that (i) meas $U \backslash \mathscr{Z}(w)<\delta$, (ii) $\sup _{U}|w|<\varepsilon /(4(1+f))$, and (iii) $\sup _{U}\left|w g^{\prime}\right|<\varepsilon / 8$. Let $V$ be an open neigh- 
borhood of $\mathscr{Z}(w)$ such that $\bar{V} \subset U$. Choose a continuous function $F$ such that $F=1$ on $\bar{\nabla}, F=0$ outside $U$, and $0 \leqq F \leqq 1$ everywhere. Let $h=F f^{\prime}+(1-F) g^{\prime}$. Then $h$ is continuous, $h=f^{\prime}$ on $\bar{V}, h=g^{\prime}$ outside $U$, and $|h| \leqq\left|f^{\prime}\right|+\left|g^{\prime}\right|$ everywhere off $\mathscr{Z}(w)$. Let

$$
G(x)=g(0)+\int_{0}^{x} h(t) d t .
$$

Then $G$ is in $C^{1}[0,1]$, and it is routine to verify that $\|g-G\|_{w}<\varepsilon$. The proof is complete since $C^{1}[0,1] \subseteq C^{1}(w)$.

THeORem 8. $C^{1}(w)=A C_{w}$ if and only if $1 / w \in L^{1}[0,1]$.

Proof. Assume that $1 / w \in L^{1}[0,1]$, and let $M=\max \left\{1, \int_{0}^{1}|1 / w|\right\}$. Then $\left\|f^{\prime}\right\| \leqq M\|f\|_{w}$ for each $f \in C^{1}[0,1]$ where $\|\cdot\|$ ' is the standard norm on $A C[0,1)$. Hence $C^{1}(w) \subseteq A C[0,1]$, and this in turn implies that $C^{1}(w)=A C_{w}$ by Lemma 7 .

Now assume that $C^{1}(w)=A C_{w}$. Hence, $C^{1}(w) \subseteq A C[0,1]$ and there exists a constant $M>1$ such that $\|f\|^{\prime} \leqq M\|f\|_{w}$ for all $f \in C^{1}(w)$. Therefore,

$$
\|f\|+\int_{0}^{1}\left|f^{\prime}\right| \leqq M\left(\|f\|+\left\|w f^{\prime}\right\|\right), \quad f \in C^{1}[0,1] .
$$

We may assume that $w \geqq 0$. For each positive integer $n$, let $v_{n} \in C[0,1]$ be defined by $v_{n}=\min \{1 / w, n\}$ and let $s_{n}=\int_{0}^{1} v_{n}$. Then $s_{n} \rightarrow+\infty$ if and only if $1 / w$ is not in $L^{1}[0,1]$. We claim that there exist $\left\{u_{n}\right\} \subset C[0,1]$ such that $\left|u_{n}\right| \leqq v_{n}$,

and

$$
\int_{0}^{1}\left|u_{n}\right| \geqq s_{n}-1
$$

$$
\left|\int_{0}^{x} u_{n}(t) d t\right| \leqq 1
$$

for $x \in[0,1]$ and all $n$. To prove this, fix $n$, and let $k$ be some integer larger than $s_{n}$. Subdivide $[0,1]$ into $k$ successive disjoint intervals $I_{1}, \cdots, I_{k}$ such that

$$
\int_{r_{1}} v_{n}=s_{n} / k
$$

Let $w_{n}$ be defined by $w_{n}(x)=(-1)^{j+1} v_{n}(x)$ if $x \in I_{j}$, and choose a continuous function $u_{n}$ with the same sign as $w_{n}$ such that $\left|u_{n}\right| \leqq\left|w_{n}\right|=v_{n}$, and

$$
\int_{I_{j}}\left|u_{n}\right|=\int_{I}\left|u_{n}\right| \geqq\left(s_{n}-1\right) / k \text {, }
$$

for $j, m=1, \cdots, k$. It is clear that $\left\{u_{n}\right\}$ satisfy the claim. Let $f_{n} \in C^{1}[0,1]$ be defined by $f_{n}(y)=\int_{0}^{y} u_{n}(t) d t$. Then $f_{n}^{\prime}=u_{n}$; hence, $\left\|w f_{n}^{\prime}\right\|=\left\|w u_{n}\right\| \leqq\left\|w v_{n}\right\| \leqq 1$. 
Furthermore, by the claim, $\left\|f_{n}\right\| \leqq 1$ and

$$
\int_{0}^{1}\left|f_{n}^{\prime}\right| \geqq s_{n}-1 \text {. }
$$

Substituting in $\left(^{*}\right)$ gives that $s_{n} \leqq 2 M+1$. Hence $\left\{s_{n}\right\}$ is bounded, and thus $1 / w \in L^{1}[0,1]$.

We now give an example of a large class of functions $w$ such that $C^{1}(w)$ properly contains $A C_{w}$.

EXAMPLE. If $w \in C[0,1]$ and $w^{\prime}\left(x_{0}\right)=0$ at some point $x_{0} \in \mathscr{Z}(w)$, then $C^{1}(w)$ properly contains $A C_{w}$. This is easily seen since, by using the definition of $w^{\prime}\left(x_{0}\right)=0$, one can show that $1 / w$ is not in $L^{1}[0,1]$.

We shall give a theorem guaranteeing that under certain very general conditions on $\mathscr{Z}(w), C^{1}(w)=C_{w}^{1}$. First, we discuss this problem. In what now follows, we use the result of Theorem 1 that $C^{1}(w)$ is a regular algebra. Suppose that $f \in C_{w}^{1}$. Then $f \in C^{1}(w)$ if and only if $f$ locally belongs to $C^{1}(w)$ at each point of $[0,1]$. Let $\mathcal{N}(f)$ be the set of all points of $[0,1]$ at which $f$ locally belongs to $C^{1}(w)$, and $\mathscr{S}(f)=[0,1] \backslash \mathcal{N}(f)$. Then $f \in C^{1}(w)$ if and only if $\mathscr{S}(f)$ is empty. But $f$ is continuously differentiable in some neighborhood of each point of $[0,1] \backslash \mathscr{Z}(w)$. Hence $[0,1] \backslash \mathscr{Z}(w) \subseteq \mathscr{N}(f)$. Furthermore, it is easily seen that any continuous function on $[0,1]$ locally belongs to $C^{1}(w)$ at each point of the interior of $\mathscr{Z}(w)$. Thus the interior of $\mathscr{Z}(w)$ is contained in $\mathscr{N}(f)$. Hence, $\mathscr{P}(f)$ is a closed subset of the boundary of $\mathscr{Z}(w)$.

LEMMA 9. If $x_{0} \in[0,1]$ and if $f \in C_{w}^{1}$ belongs locally to $C^{1}(w)$ at each point of $[0,1] \backslash\left\{x_{0}\right\}$, then $f \in C^{1}(w)$.

Proof. (We give the proof in case $x_{0}=0$; from this one can see how to proceed in the other cases.) Let $g \in C_{w}^{1}$ belong locally to $C^{1}(w)$ at each $y \in(0,1]$, and let $\varepsilon>0$. Choose $f \in C^{1}[0,1]$ and $a, c \in(0,1)$ such that (i) $\|f-g\|<\varepsilon / 12$, (ii) $a<c$, (iii) $\sup _{y \in[0, c]}\left|\left(w g^{\prime}\right)(y)\right|<\varepsilon / 12$, (iv) $\sup _{y \in[0, c]}|w(y)|<\varepsilon / 12\left(\left(1+\left\|f^{\prime}\right\|\right)\right.$ ), and $(v)$

$$
\int_{0}^{c}\left|f^{\prime}\right|<\varepsilon / 12 \text {. }
$$

But $g \mid[a, 1]$ is an element of the Banach algebra of restrictions of functions in $C^{1}(w)$ to $[a, 1]$ (where the norm of such a function is the infimum of the norms of functions in $C^{1}(w)$ agreeing with it on $\left.[a, 1]\right)$. Hence, since the $C^{1}$-functions are also dense in this restriction algebra, there exists $h \in C^{1}[a, 1]$ such that (vi)

$$
\sup _{y \in[a .1]}|h(y)-g(y)|+\sup _{y \in[a 1]}\left|\left(w h^{\prime}\right)(y)-\left(w g^{\prime}\right)(y)\right|<\varepsilon / 12 .
$$

Let $b, a<b<c$, be chosen so that (vii) $\int_{h}^{c}\left|h^{\prime}\right|<\varepsilon / 12$. Finally, choose $\phi \in C[0, c]$ such that (viii) $\phi(c)=h^{\prime}(c)$, (ix) $|\phi(y)| \leqq\left|f^{\prime}(y)\right|$ for $0 \leqq y \leqq b$, and 
(x) $|\phi(y)| \leqq\left|h^{\prime}(y)\right|$ for $b \leqq y \leqq c$. Let $\psi(y)=\phi(y)$ for $0 \leqq y \leqq c$, and $\psi(y)=h^{\prime}(y)$ for $c \leqq y \leqq 1$, and define

$$
g^{*}(y)=f(0)+\int_{0}^{y} \psi(t) d t .
$$

Then $g^{*} \in C^{1}[0,1]$, and it is routine to check that $\left\|g-g^{*}\right\|_{w}<\varepsilon$. The proof is complete.

LEMMA 10. If $f \in C_{w}^{\mathbf{1}}$, then $\mathscr{S}(f)$ is a perfect set.

Proof. Suppose that $x_{0}$ is an isolated point of $\mathscr{S}(f)$. Then there is a neighborhood of $x_{0}$ in which $f$ locally belongs to $C^{1}(w)$ at each point except $x_{0}$. But we may assume that $f$ locally belongs to $C^{1}(w)$ at each point of $[0,1] \backslash\left\{x_{0}\right\}$. (If not, one can find an element in $C_{w}^{1}$ agreeing with $f$ in a neighborhood of $x_{0}$ for which it is true.) Then $f$ must belong to $C^{1}(w)$ at $x_{0}$ by Lemma 9 , and this contradiction proves the lemma.

The following theorem is clear from Lemma 10 , the discussion preceding Lemma 9, and Theorem 120 of Sierpinski (1952).

THEOREM 11. If the boundary of $\mathscr{Z}(w)$ is countable, then $C^{1}(w)=C_{w}^{\mathbf{1}}$.

The result of Theorem 11 and its proof are analogous to a theorem of Ditkin (see p. 226 of Naimark (1964)), although $C^{1}(w)$ is not an ideal in $C_{w}^{\mathbf{1}}$. In addition, because there are nontrivial point derivations on $C_{w}^{1}$ at points where $w$ is not zero, we see that Ditkin's condition will not hold in $C_{w}^{1}$ unless $w$ is identically zero.

Corollary 12. If $\tilde{D}_{w}$ is almost injective, then $C^{1}(w)=C_{w}^{1}$.

PROOF. This follows from Theorem 11 and Theorem 6.

Theorem 11 says that $C^{1}(w)=C_{w}^{1}$ except possibly when $\mathscr{Z}(w)$ is a "complicated" set. We now give such an example where $C_{w}^{1} \neq C^{1}(w)$.

EXAMPLE. Let $\mathscr{T}$ be the Cantor ternary set on $[0,1]$, and let $w$ be defined by $w(y)=(\text { distance }(y, \mathscr{T}))^{1 / 3}$. Then $1 / w \in L^{1}[0,1]$; hence, by Theorem 8 , $C^{1}(w)=A C_{w}$. But since $C_{w}^{1}$ contains the Cantor ternary function, we see that $C^{1}(w) \neq C_{w}^{1}$.

\section{References}

R. J. Loy (1970), 'Maximal ideal spaces of Banach algebras of derivable elements', J. Austral. Math. Soc. 11, 310-312.

M. A. Naimark (1964), Normed rings (Noordhoff, Groningen, The Netherlands, 1964).

C. E. Rickart (1960), General theory of Banach algebras (Van Nostrand, New York, 1960).

W. Sierpinski (1952), General topology (University of Toronto Press, Toronto, 1952).

Department of Mathematics

University of Nevada, Las Vegas

Las Vegas, Nevada 89154, U.S.A. 